mind will tend to prevent the student from forming any clear and distinct conception of the phenomena.

Let us now examine how far M. Voipicelli's experimental skill and extensive reading have enabled him to give an accurate account of the phenomena, and how far he may have fallen into error from not availing himself of the idea of electric potential, but continuing to employ that of latent electricity.

Melloni, in his exposition, bas represented the homonymous electrification $(\beta)$ as greater on the side of the induced body further from the inducior. The fact, however, is that the electrification is distributed in the same way as it would be if the inductor were in its actual position and insulated, but without charge. It will therefore be densest on the projecting parts of the induced body; but if the two extremities of this body are geometrically similar, and if the inductor is made of a conducting substance, it will be somewhat denser on the extremity (B) next the inductor, because the surface of the inductor itself (c) will become electrified, and the electricity on the side next to $B$ will be negative.

But the inequality of the distribution of the negative electrification $(\alpha)$ is so much greater that it completely masks that of $(\beta)$, so that from an experimental point of view we must regard this error of Melloni as a very trifling one.

The next point we must notice is the mode in which objection (3) is expressed. It is as follows:-

"(3) Because of the two kinds of electricity. which coexist upon the induced insulated body, only the homonym of the inductor is dissipated by contact with the air." (The italics are our ownl.)

We have no evidence whatever that electricity is ever dissipated by contact with air, whether dry or moist, unless the electric density is so great that a disruptive discharge takes place in the forms of "glow," "brush," or "spark," from sharp points connected with the electrified body.

If the electrified body and the surrounding conductors have rounded surfaces, and if the potential is moderate, it appears from the experiments of Boltzmann ${ }^{1}$ that no measureable quantity of electricity passes through air or other gases, even when greatly rarefied, and when the experiment is continued for fourteen hours.

I have myself been unable to detect any conduction through a stratum of still air of two millimetres thickness, even when the temperature was raised to a red heat, and when steam, or the vapour of mercury or of sodium was introduced between the oppositely electrified surfaces. If, however, smoky air was intro. duced, there was a considerable effect arising from convection by the solid particles.

The cause of the powerful electrical effects of the stream of heated malter rising from a Bunsen's burner or from a red-hot ball, as in Gutbrie's experiments, requires a special investigation.

The dissipation of the charge of insulated bodies which we actually observe seems to depend principally on the insulating supports on which they are placed, and if these are of good glass the conduction is almost entirely due to moisture on the surface of the glass. If the air which is in contact with the glass insulator is perfectly dry the dissipation of electricity will be extremely small, eren when the air in contact with the electrified body itself is loaded with moisture.

It is not, therefore, by contact with the air that the electricity escapes, but by conduction to the earth along the so-called insulating supports, and the effect of this conduction is of course to reduce the potential to zero by discharging electricity of the same kind with that of the inductor.

We come next to the fourth of the five facts mentioned under the head of the First Experiment. It is stated as follows :-

" 4 . Points applied to the extremity of the cylinder nearest to the inductor allow only the homonym of the inductor to escape, and not at all the opposite electricity."

This will be the case if the point is electrically connected with the earth, and made to apfroach any part of the surface of the cylinder; but if, as the words seem rather to imply, the point is at ached to the cylinder and projects into the air, then the statement is exactly opposite to that given by Riess in Art. 247 of his book, who correctly tells us that if the cylinder has a sharp point at one end, then if the point is turned towards the inductor, the cylinder becomes charged similarly to the inductor, whereas if the point is tumed away frum the inductor, the cylinder becomes charged oppositely to the inaluctor, the discharge from the point being always of that kind of electricity which exists on the part of the cylinder where the point is placed.

The fifth fact stated to be established by the experiment is-

" 5. Induced electricity of the first kind (opposite to that of the inductor) is not transferred from the induced body to the in. ductor, but the electricity of the inductor may certainly be transferred to the induced body."

For the sake of distinctness, let us say that the inductor is positive, then it is here asserted that negative electricity does not pass from the cylinder to the inductor, but that positive electricity passes from the inductor to the cylinder.

If M. Volpicelli can give us an experimental method of distinguishing between the passage of negative electricity from $B$ to $A$, and the passage of positive electricity from $A$ to $B$, we may expect to learn more of the nature of electricity than any of our physicists have hitherto even hoped for.

J. Clerk Maxwell.

\section{Cherry Blossoms}

IN the last number of NaTURE (vol. xiv., p. Io), Mr. Pryor states that the flowers of the wild cherry are bitten off in large numbers in much the same manner as I formerly described in the case of the primrose. Some days ago I observed many cherry blossoms in this state, and to-day I saw some actually falling. I approached stealthily so as to discover what bird was at work, and behold it was a squirrel. There could be no doubt about it for the squirrel was low in the tree and aciually had a blossom between its teeth. It is none the less true that birds likewise bite the flowers of the cherry tree.

Down, Beckenbam, May 6

Charles Darwin

\section{The Pollen of the Cherry}

THE practice of the indefinite reproduction of woodcuts by means of clichés has frequently given rise to the repetition of erroneous drawings in one scientific text-book after another. Botanical text-books seem to have suffered especially in this way, in consequence of the great dearth of new and original illustrations by which they are characterised. Many botanical students must have been puzzled by the peculiar appearance presented by the pollen of the cherry in a very familiar drawing. It is hardly sufficiently explained that "the escape of the fovilla in an irregular jet," as there represented, has nothing to do with the process of fertilisation, but is an altogether abnormal pheno. menon depending on the bursting of the pollen-grain from artificial moistening. The shape of the pollen-grain, as drawn, for example, in Balfour's "Class-book of Botany," Le Maout and Decaisne's "General System of Botany," and Dr. Hooker's Science Primer "Botany" 1 is also incorrectly indicated. The perfectly spherical form represented in these drawings is almost, if not altogether, confined to anemophilous plants, fertilised by the wind. The cherry is, on the contrary, entomophilous, and its pollen partake of the general character of this class of platis.
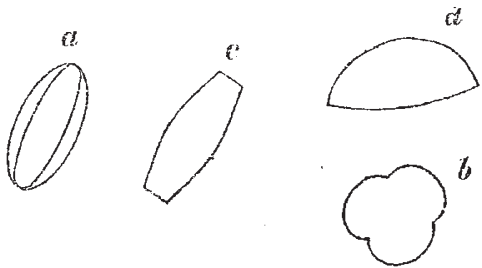

Though somewhat variable in size and form, the grains are, I believe, never spherical, but ellipsoidal, with three longitudinal furrows, as represented in the longitudinal and apical aspects, $a, b$, in the accompanying figure. The pollen has, however, well-marked characters of its own, which distinguish it from that of allied plants, the ends often appearing truncated, as represented in $c$, and some or all of the grains more gibbous on one face than another $(d)$. Most pollen-grains assume a more spherical form on being moistened with water.

\section{Alfred W. Bennett}

I In Hooker's Primer there is the further complication of the accidental transposition of the figures of the cherry and evening-primsose, the well: known triąngular form of the latter being attributed to the former. 